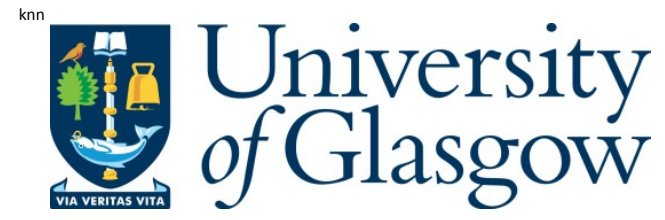

O 'Shea, K ., R ode, K., K urt, H., M cGrouther, D., and M acL aren, D.A. (2015) Concentric $360^{\circ}$ domain wall nesting in magnetic tunnel junction films: a Lorentz TEM study. Journal of Physics D: A pplied Physics, 48 (5). 055001. ISSN 0022-3727

Copyright @ 2015 IOP Publishing

http://eprints.gla.ac.uk/101522/

Deposited on: 20 J anuary 2015

Enlighten - Research publications by members of the University of Glasgow http://eprints.gla.ac.uk 
This is an unedited version.

For a full text copy, please go to doi:10.1088/0022-3727/48/5/055001 


\title{
Concentric $360^{\circ}$ domain wall nesting in magnetic tunnel junction films: A Lorentz TEM study
}

\author{
K J O'Shea ${ }^{1}$, K Rode ${ }^{2}$, H Kurt ${ }^{2}$ and D A MacLaren ${ }^{1}$ \\ ${ }^{1}$ SUPA, School of Physics and Astronomy, University of Glasgow, G12 \\ 8QQ, UK \\ ${ }^{2}$ School of Physics and CRANN, Trinity College, Dublin 2, Ireland
}

\begin{abstract}
We describe the formation of an unusual concentric magnetic domain wall pattern in the free layer of a bottom pinned magnetic tunnel junction. Lorentz microscopy reveals that repeated switching of the free layer with a magnetic field applied perpendicular to the exchange bias direction can produce a series of concentric $360^{\circ}$ domain wall loops, a phenomenon we refer to as domain wall nesting. We propose two necessary ingredients for the behaviour: (i) inhomogeneities in the grain-by-grain magnetic dispersion that break local symmetry to produce a preferential sense of magnetic rotation upon field switching; and (ii) structural defects that act to pin $360^{\circ}$ domain walls. Further control of this behaviour may provide new functionality for future device applications.
\end{abstract}

\section{Introduction}

Magnetic tunnel junctions (MTJs) with $\mathrm{MgO}$ tunnel barriers are of interest as spin-torque magnetoresistive random-access-memory (ST-MRAM) devices [1, 2], magnetic sensors [3, 4] and logic elements [5,6]. They require reproducible control of the magnetisation of the individual magnetic layers [7,8]: for ST-MRAM storage cells, the magnetisation directions must be switched between a parallel and anti-parallel alignment; whilst for magnetic sensors, all relative magnetisation orientations may be required. A uniform reversal mechanism of the free magnetic layer is desirable to ensure reliable MTJ devices, however pinning of discrete regions of magnetisation by topological features or defects is known to impair performance. In particular, the formation of $360^{\circ}$ domain walls (DWs) has been reported for MTJ [9-11] and related exchange bias systems [12-16] and is known to disrupt uniform switching, leading to noise and stability issues during device operation. Conceptually, these $360^{\circ} \mathrm{DWs}$ arise from the coalescence of two of the more common $180^{\circ}$ Néel DWs that separate regions of opposite magnetisation [17,18], often because the first $180^{\circ}$ DW becomes pinned by a structural defect. Interaction with a second $180^{\circ} \mathrm{DW}$ of the same chirality then produces the $360^{\circ} \mathrm{DW}$, which commonly exist as line defects that are topologically robust and difficult to remove by field switching. They can remain stable even in fields of several tens of $\mathrm{mT}$ as a result of the energy barrier associated with unwinding the wall, which arises from the strong attractive magnetostatic interaction between the constituent $180^{\circ}$ DWs [19]. Such DWs have frequently been observed in multilayer films, where their behaviour is modulated by issues of 
interlayer coupling, layer coercivity and anisotropy dispersion [18]. More complex $360^{\circ} \mathrm{DW}$ structures have also been reported, for example, where the two $180^{\circ}$ Néel walls curl around to form a closed $360^{\circ} \mathrm{DW}$ loop, or where the DWs are constrained with patterned nanostructures such as rings [20-24], ellipses [9,11] and nanowires [25-27], in which cases the element edges play a key role in DW nucleation. The formation of $360^{\circ} \mathrm{DWs}$ is generally undesirable, although they can reduce the switching current in favourable cases [21]. From a more fundamental perspective, the study of topological spin structures has undergone a renaissance in recent years, particularly with regards to the collective behaviour of soliton lattices, skyrmions and vortices, much of which are only observable at cryogenic temperatures [28]. Here, we study the room-temperature nucleation and self-organisation of concentric $360^{\circ} \mathrm{DW}$ loops in MTJ continuous films, which we refer to as DW nesting. We explore this effect by applying magnetic fields both parallel and perpendicular to the exchange bias direction and find that the behaviour is sensitive to both the field direction and sample history, the latter of which could be useful as a new type of magnetic counter [29] or memory element. By performing elemental analysis, we observe local intermixing across the tunnel barrier in some locations, causing enhanced ferromagnetic coupling between the MTJ magnetic layers. When patterned into small elements, this effect is likely to perturb the operation of MTJ devices, or introduce considerable variation in behaviour across an array of elements.

\section{Experiment}

A conventional MTJ stack [30] was sputter-deposited onto SiN windowed substrates for planview magnetic imaging [31]; a schematic of the stack structure, hereafter denoted S2, is given in the inset of figure 1(a). A CoFeB/MgO/CoFeB stack forms the active part of the MTJ, the uppermost layer forming the 'free' or 'sensing' layer with a magnetisation that is free to rotate under an applied field whilst the lower layer is pinned by the $\mathrm{CoFe} / \mathrm{Ru} / \mathrm{CoFeB}$ synthetic antiferromagnet (SAF). Ta layers were deposited for use as electrodes and a $\mathrm{NiFe}$ layer was used as a seed to introduce (111) texture, the magnetisation of which is pinned by a thin IrMn layer. (Layer thicknesses are indicated in the figure). The bottom and top electrodes were deposited in a high vacuum chamber with a base pressure of $1 \times 10^{-7}$ Torr. After the deposition of bottom electrodes, the wafer was transferred in vacuum to a UHV chamber with a base pressure of $2.1 \times 10^{-10}$ Torr to deposit the active layers $(\mathrm{CoFeB} / \mathrm{MgO} / \mathrm{CoFeB})$. The films were annealed at $350^{\circ} \mathrm{C}$ for 1 hour in an in-plane magnetic field of $0.8 \mathrm{~T}$ to crystallize the $\mathrm{CoFeB}$ layers and to set an in-plane easy axis. A simplified reference stack was also deposited with the following structure $\mathrm{SiN} / \mathrm{Ta}(5) / \mathrm{Co}_{40} \mathrm{Fe}_{40} \mathrm{~B}_{20}(3) / \mathrm{MgO}(2.5) / \mathrm{Ta}(5)$, hereafter referred to as $\mathrm{S} 1$, where the numbers in brackets are the layer thicknesses in $\mathrm{nm}$. 
Magnetic characterisation and tunnelling magnetoresistance (TMR) measurements were conducted using separate samples that were deposited onto Si substrates. The physical microstructure was then observed by transmission electron microscopy (TEM) using an FEI Tecnai F20. The magnetic reversal behaviour of the MTJ was investigated by the Fresnel mode of Lorentz TEM, performed in a Philips CM20 FEG instrument [32]. An in-plane magnetic field was applied to samples by tilting them within the field produced by the weakly-excited objective lens of the microscope. Note that this tilting results in image shifts that must be corrected manually and inevitably means that the field of view shifts slightly from image to image in the results presented here.

In addition to viewing the MTJ in plan-view, cross-sections were fabricated for subsequent imaging and chemical analysis of the constituent layers and interfaces. These were milled using standard protocols [33] on an FEI Nova Dualbeam Focused Ion Beam (FIB) system that was operated at $30 \mathrm{kV}$, concluding with a final $5 \mathrm{kV}$ polishing stage. Elemental analysis was carried out in a JEOL ARM 200cF scanning transmission electron microscope (STEM) using a probe half angle of $22 \mathrm{mrad}$. Electron energy loss spectroscopy (EELS) data were recorded with a Gatan Quantum 965 spectrometer with a collection half angle $(\beta)$ of $72 \mathrm{mrad}$ and a dispersion of $0.5 \mathrm{eV} /$ channel. The spectrum imaging plug-in of Gatan Digital Micrograph was used to record spectrum images across the $\mathrm{CoFeB} / \mathrm{MgO} / \mathrm{CoFeB}$ interface region using a step size of $0.25 \mathrm{~nm}$ and $2.0 \mathrm{~s}$ acquisition time per pixel. The thickness of the polished cross section was measured from the EELS data to be of order $70 \mathrm{~nm}(\mathrm{t} / \lambda \sim 0.6-0.8)$.

\section{Results}

\subsection{Magnetoresistance}

TMR curves for a typical S2 stack are given in figure 1(a) [30], where the arrows indicate the parallel and anti-parallel configurations of the two $\mathrm{CoFeB}$ layers. The external magnetic field was aligned along an easy axis direction. Upon reducing the magnetic field from a large positive value, the relative alignment of the free and pinned $\mathrm{CoFeB}$ layers changes from parallel to anti-parallel close to zero field (indicated by labels (i) and (ii) in figure 1(a)). This corresponds to a considerable TMR ratio, in excess of $240 \%$ at room temperature. With increasing negative applied field, the TMR ratio reduces as the lower layer is slowly rotated away from the orientation set by the underlying SAF. Complete layer rotation and parallel alignment is achieved at a field of $\sim 100 \mathrm{mT}$ (indicated by (iii) in figure 1(a)). Figure 1(b) shows a plan-view bright field image of sample S2, the full MTJ stack, with a diffraction pattern inset. It should be noted that the plan-view images and diffraction patterns arise from a projection through the complete multilayer stack. The contrast observed is consistent with a 
polycrystalline structure comprising small randomly oriented grains, confirmed by the ring diffraction pattern inset. A cross-sectional bright field image is given in figure 1(c), where the $\mathrm{MgO}$ barrier appears as a bright band of intensity across the image. Some degree of layer roughness can be seen and occurs on a lengthscale of 20-30 nm. A Fourier transform of the image shows a set of sharp spots indicating high-quality epitaxial growth across the barrier.

\subsection{Lorentz microscopy: image interpretation}

In order to aid interpretation of the magnetic images presented throughout the remainder of this paper, a schematic illustration of a typical $360^{\circ} \mathrm{DW}$ is given in figure 2(a). Moving from left to right across the film, spins rotate clockwise in the plane of the film and the $360^{\circ} \mathrm{DW}$ consists of two coupled $180^{\circ}$ Néel DWs. The relative orientation of spins spanning each $180^{\circ}$ DW determines the wall chirality (rather than the orientation of the core itself); here both walls have a clockwise (cw) rotation of spins from left to right, defining both as having positive chirality. In this case, the magnetisation of the central domain is constrained by the regions of opposing magnetisation on either side, which present a large energy barrier to unwinding. In contrast, if the chirality of either wall was reversed, then the central domain can easily rotate, thereby annihilating both walls. Figure 2(b) illustrates the observed contrast in the Fresnel mode of Lorentz microscopy, where a Lorentz deflection of the electron beam passing through the sample gives rise to distinctive dark or bright bands of electron intensity at the DW locations if the main imaging lens is defocused by a distance $\Delta z$. Note that the bright or dark regions of increased or decreased intensity arise from the interference of electron beams transmitted through the domains, and is therefore independent of the spin structure or chirality of the DW itself: these are inferred from observation of the DW evolution, as outlined below. Additionally, it must be noted that for the polycrystalline continuous films investigated here, weak magnetic contrast also arises as a result of the random orientation of the anisotropies of individual crystallites, producing 'magnetisation ripple', which is illustrated schematically within the magnetic domains in figure 2(b). The ripples lie perpendicular to the mean direction of magnetisation, so it is possible to deduce the magnetisation direction by taking Fourier transforms of the magnetic images.

\subsection{Lorentz Microscopy: Stack S1}


We now turn to the Lorentz microscopy results. Magnetic reversal of the full MTJ stack is better understood by first introducing the magnetic reversal behaviour of sample S1, a single $\mathrm{CoFeB}$ layer. Figure 3 shows a Fresnel image sequence that summarises the magnetic reversal of S1 within a magnetic field aligned along both an easy axis (a-d) and hard axis (e-g). Arrows in the images indicate the magnetisation direction and were deduced from Fourier transforms of the images, taking ripples to lie perpendicular to the local magnetisation as described above. Under the influence of a hard axis (HA) field, figures 3(a)-(d), reversal occurs via smooth, uniform rotation of the magnetisation, as expected for a single ferromagnetic film. It may be observed that the ripple contrast is greater at lower fields, figures 3(b) and 3(c), as the result of a larger magnetisation dispersion. In contrast, reversal under a field applied along the easy axis (EA) shows a much more rapid switching behaviour, similar to the rapid TMR change seen in Figure 1: images are given in figures 3(e)-(h), which were collected after several field sweeps. Two DW sections formed during a previous field cycle are identified (figure $3(\mathrm{e})$ ) as $360^{\circ} \mathrm{DWs}$ from the characteristic parallel black and white lines. The separation of the twin $180^{\circ}$ Néel DWs producing these lines is determined by a balance of the magnetostatic energy that attracts the walls together; the exchange interaction that pushes them apart; and a competing Zeeman energy term that acts to minimise the number spins lying anti-parallel to an external field. Thus, as the field was reduced to $0 \mathrm{~T}$, the two $180^{\circ}$ Néel DWs separated slightly. A close-up view of the ends of the $360^{\circ} \mathrm{DWs}$ is given in figure 3(h) and highlights the termination of DWs on structural defects. Reversal of the magnetisation occurred abruptly at a field of $-0.32 \mathrm{mT}$, via the nucleation and growth of reverse domains which rapidly swept through the film, to leave it as in figure $3(\mathrm{~g})$. The film is predominantly single domain but residual DWs remain pinned between the two defects, in this case leaving the $360^{\circ} \mathrm{DW}$ lying perpendicular to the surrounding magnetisation.

This field reversal sequence demonstrates that coherent rotation of the spins in the main film cannot always unwind the $360^{\circ} \mathrm{DW}$ in the presence of pinning sites and that these topological spin defects are intimately connected to structural defects in the film. In this case, the appearance of these defects is consistent with a particulate in one of the layers and such physical artefacts are known to perturb the local magnetisation of the surrounding layers [34] to form $360^{\circ}$ DWs $[17,18]$. In other cases, the physical identity of the defect is less obvious. Wall formation also tends to coincide with regions of increased ripple contrast in Lorentz TEM, indicating an increased dispersion of the grain-by-grain magnetisation direction. These two features are sufficient to account for the formation of $360^{\circ} \mathrm{DWs}$, as illustrated by the onedimensional spin model sketched in figure 4(a). The figure shows a line of exchange-coupled spins with a central pinned spin that is picked out in red. Each line of the schematic then depicts the evolution of the spin configuration as an opposing external magnetic field is 
applied, with each spin's orientation constrained by a balance of Zeeman and exchange energy terms. In addition, we assume an easy axis to lie along the illustrated strip, so that the spins tend to align horizontally at remanence. In general, when a magnetic field is applied anti-parallel to a film's magnetisation, it is equally favourable for the spins to rotate clockwise (cw) or counter clockwise (ccw) to align with the field and very small perturbations or local asymmetries will be sufficient to direct the sense of rotation. In the schematic, the spins on the left of the pinned spin rotate $\mathrm{ccw}$ whilst those on the right rotate $\mathrm{cw}$. As the external field reverses, the spins first rotate to produce a $180^{\circ} \mathrm{DW}$, then continue to produce a $360^{\circ} \mathrm{DW}$ comprising a pair of $180^{\circ}$ Néel DWs of the same chirality. If the external field was reversed once more and the spins rotate back in the opposite sense, then the $360^{\circ} \mathrm{DW}$ is 'unwound' and eliminated. In general, this simple model is sufficient to explain the nucleation and annihilation of $360^{\circ} \mathrm{DWs}$ in the single film (S1) stack and experimentally we did not find any more complex spin structures during the cycling of S1 samples.

\subsection{Lorentz microscopy: stack $S 2$}

One may expect similarities between the reversal of the single CoFeB layer and that of the free $\mathrm{CoFeB}$ layer within the full MTJ stack, with the main difference being that the stack has an axis imposed by the exchange bias. Consequently, the free layer of the stack will exhibit a different coercivity to that of the S1 stack, along with an additional exchange field. Furthermore, there are numerous magnetic layers in the full MTJ system, so that the magnetic image contrast observed will be a superposition of the magnetic contrast from each of the magnetic layers in the stack. The magnetic reversal of the full stack is presented in the following sections.

Figure 5 shows a sequence of Fresnel images of the S2 MTJ stack with a magnetic field applied parallel to the exchange bias (EB) axis. Reversal of the free layer occurred via the nucleation and growth of domains that swept rapidly through the film, similar to the behaviour of stack S1 in figures 2(e)-(h). $360^{\circ}$ DWs were occasionally observed (in 3 of 10 S2 stacks) but did not dominate or disrupt the reversal behaviour. However, the ripple contrast is obviously more complex than in the $\mathrm{S} 1$ device. A high density magnetic contrast appeared when a positive field was applied (figures 5(b) and 5(h)), which we attribute to the reversal of one of the two ferromagnetic layers coupled to the IrMn (i.e. either the CoFe or the $\mathrm{NiFe}$ layer) that cannot be distinguished in projection. This reversal is similar to that seen in previous studies of exchange bias systems [10] and is not observed in the TMR loop as it does not contribute to the tunnel resistance across the $\mathrm{MgO}$ barrier. At negative fields of order $\sim 50$ $\mathrm{mT}$ (not shown), the reversal of the pinned $\mathrm{CoFeB}$ layer (i.e. from region (ii) to (iii) in figure 
1(a)) was also observed, in agreement with the TMR data. The coercivity and exchange field were extracted as $\mathrm{H}_{\mathrm{c}}=1.2 \pm 0.3 \mathrm{mT}$ and $\mathrm{H}_{\mathrm{EB}}=0.9 \pm 0.3 \mathrm{mT}$ and the behaviour observed here is consistent with that expected for a MTJ [30].

In contrast to the smooth reversal above, reversal of the free layer under a field applied perpendicular to the EB axis displayed more complex behaviour, as illustrated in Figure 6. At a field of $+16.8 \mathrm{mT}$, figure $6(\mathrm{a})$, the magnetisation of the free layer (indicated by the white arrow) was aligned parallel to the magnetic field. One might expect the magnetisation to rotate uniformly to align with the EB axis when reducing the field, analogous to the HA reversal of the S1 stack. However, in regions of stronger ripple contrast (and hence greater magnetisation dispersion), the rotation was often inhomogeneous. For example, in the image sequence of figures 6(b)-(c), the magnetisation rotated $\mathrm{cw}$ in the region denoted $\mathbf{A}$ and $\mathrm{ccw}$ in the region denoted $\mathbf{B}$, with the direction of rotation indicated by the black arrows. Consequently, anti-parallel domains were created in the free layer at remanence, figure 6(c), with the domains bounded by $180^{\circ}$ DWs. Since these domains only account for a small fraction of the film, the device would retain a net magnetisation. Note that the wall chirality can be assigned by considering that the spins at the centre of the $180^{\circ} \mathrm{DW}$ will retain the alignment of the first image in the sequence, i.e. parallel to the applied magnetic field. Note also that the DW in figure 6(c) assumed a zigzag shape, a low-energy configuration that avoids the formation of extended charged head-to-head $180^{\circ} \mathrm{DWs}$ and maximises the alignment with respect the EB direction, at the expense of a slightly increased wall length [34, $35]$.

Continuing the field sweep, a first $360^{\circ} \mathrm{DW}$ nucleates between figures 6(c) and 6(d), initiated by a very small negative field when a region of the anti-parallel domain (labelled $\mathbf{C}$ ) suddenly switches magnetisation direction by $180^{\circ}$. The process is similar to that seen in the entire film when the external field is aligned along the EB axis. We can infer that the magnetisation of the small region, bounded to the right by a $360^{\circ}$ and to the left by a $180^{\circ} \mathrm{DW}$, must have continued to rotate $\mathrm{ccw}$ since a $\mathrm{cw}$ rotation would have unwound the DW lying to the right. Such rapid switching of a local area was common to the formation of $360^{\circ}$ DWs in S2 films. Once nucleated, the switched region continues to grow and the $180^{\circ} \mathrm{DW}$ propagates throughout the anti-parallel region (figure 6(d), region B) until in figure 6(e) a $360^{\circ} \mathrm{DW}$ loop (denoted DWL1) is formed, the magnetisation consistently reversing in a ccw sense. Thus, the images can be interpreted as sketched in the model section of DW in figure 4(a), the film on either side of the $180^{\circ} \mathrm{DW}$ rotating to generate two walls of the same chirality that together from a $360^{\circ} \mathrm{DW}$. The DW is observed experimentally by the characteristic black and white regions of increased or decreased intensity, which is strongest in wall sections that lie parallel to the surrounding magnetisation, consistent with the description of figure 2 . Note that the 
DW loses its zigzag form since head-to-head walls need no longer be minimised. Such $360^{\circ}$ DW loops have been seen previously and are known to be more stable than isolated straight $360^{\circ}$ wall sections because the energy barrier associated with unwinding the wall is higher [15].

The last stage of the field reversal sweep is to increase the applied field back to a large positive value (figures 6(e)-(i)), after which the $360^{\circ}$ domain wall loop, DWL1, remains. The reason for the retention, rather than unwinding of DWL1 can be understood by considering the direction of the magnetisation rotation throughout the image sequence. The region within DWL1 continues to rotate ccw, as indicated by the black arrows in each image, rather than reversing direction. Outside the loop, on the other hand, the direction of rotation reverses. A conceptual understanding of this process is provided by the 1 dimensional model of figure 4(b), which follows the same scheme as figure 4(a) and concludes with a change in DW contrast, as observed experimentally (consider, for example, the DW section marked by a yellow line in figures 5(e) and 5(i)). In essence, rotation of the magnetisation as indicated developed a new $180^{\circ} \mathrm{DW}$ inside DWL1 and removed the outer $180^{\circ} \mathrm{DW}$.

Following the field sweep described above, a Fresnel image sequence of the same area during a second field sweep is presented in figures 6(j)-(n) and is more interesting. Figure 6(j) follows immediately after figure 6(i) and the contrast changes are similar to those seen in the first field sweep (figures $6(\mathrm{a})$,(b)) but with a $360^{\circ} \mathrm{DWL}$ already present in the film. Once again, ripple contrast increased and became inhomogeneous, and magnetisation in the region denoted A rotated cw whilst region B rotated ccw. The two $180^{\circ}$ DWs of DWL1 separated slightly at remanence (figure 6(1)), adopting a ragged zigzag profile as before. A further $180^{\circ}$ DW developed at remanence, splitting region $\mathbf{B}$ into two regions, denoted $\mathbf{B}$ and $\mathbf{C}$ in figure $6(\mathrm{l})$. Continuing to a small negative field, the evolution from figures $6(\mathrm{l})$ to $6(\mathrm{~m})$ copied that between figures $6(\mathrm{c})$ and $6(\mathrm{~d})$, with a $360^{\circ} \mathrm{DW}$ arising from the sudden reversal of an isolated area. The area switched from being aligned anti-parallel to region $\mathbf{A}$ in figure 6(1) to parallel in $6(\mathrm{~m})$ and we note that physically, the area is close to that which switched suddenly during the previous field sweep. This area expanded as before, yielding a second $360^{\circ}$ DWL nested inside the original (figure 6(n)). The one-dimensional model corresponding to this stage is presented in figure 4(c), where it is demonstrated that a consistent rotation of magnetisation in one direction is sufficient to develop new DWLs. Upon each field cycle, DWL1 oscillated in black-white contrast as $180^{\circ} \mathrm{DWs}$ develop and were eliminated, simultaneous with new $360^{\circ}$ DWs forming in the region of preferential rotation. The process could be repeated multiple times, with each field sweep producing a further $360^{\circ}$ DWL. In a different region of the film, we found it possible to nucleate 7 nested $360^{\circ}$ DWLs after 7 consecutive field cycles, as shown in figure 6(o). These DWLs were found to be very stable, persisting to fields much 
greater than those required to reverse the film, and requiring fields of up to $\sim 90 \mathrm{mT}$ for their elimination.

\subsection{Electron energy loss spectroscopy}

We now turn to consider the nature of DW pinning and the origins of the magnetic dispersion and focus on the prospect of defects in the barrier oxide layer. As there was a degree of visible roughness to the tunnel barrier layer, electron energy loss spectrometry (EELS) was carried out to characterise the degree of intermixing and assess the possibility of ferromagnetic bridges existing across the $\mathrm{MgO}$ barrier. A summary of the results is given in figure 7. Two separate regions, with apparent differences in layer roughness, were chosen for elemental analysis and are illustrated in the high angle annular dark field STEM images of figures 7 (c) and (d), where the tunnel barrier appears dark whilst heavier elements ( $\mathrm{Co}, \mathrm{Fe})$ appear bright. EELS spectra were acquired within the red boxes of each STEM image and summed along the direction parallel to the interface to improve signal statistics. Figures 7(a) and (b) show the extracted variation in (normalised) intensities of the O-K edge, $\mathrm{Fe}_{2,3}$-edge and the $\mathrm{Co}_{2,3}$-edge in the two regions, with the data corrected for plural scattering effects [36] using Fourier-ratio deconvolution [37]. The two data sets clearly differ in the quantity of $\mathrm{Fe}$ and Co that is apparent throughout the barrier layer. Dashed vertical lines indicate where the $\mathrm{O}$ intensity falls to half the maximum value, roughly indicating the boundary between the $\mathrm{MgO}$ layer and the adjacent $\mathrm{CoFeB}$ layers. It is worth noting that as this specimen is a crosssection, the images and data arise from 2-dimensional projections of 3-dimensionally rough interfaces. Thus, abrupt interfaces are not expected in the EELS profiles and there is some overlap of the $\mathrm{O}$ and $\mathrm{Co} / \mathrm{Fe}$ signals in a $\sim 2 \mathrm{~nm}$ wide region at the interfaces in figure $7(\mathrm{~b})$, in reasonable agreement with the observed layer roughness and therefore not an indicator of true intermixing. In figure 8(a), however, the Co and Fe signals do not reach zero intensity within the oxide layer and intermixing is clearly significant. This large degree of overlap of the elemental profiles indicates either direct contact of the $\mathrm{CoFeB}$ layers through pinholes in the oxide barrier or at least regions of increased proximity of the two layers and thus of enhanced ferromagnetic coupling across the barrier. Either effect could be expected to alter device behaviour during field sweeps.

\section{Discussion and conclusions}

An essential requirement for the formation of nested DWLs is the magnetisation's preferential rotation direction within certain regions of the free layer; in the images presented here, the region enclosed by DWL1 consistently rotated $\mathrm{ccw}$, whilst the external region rotated $\mathrm{cw}$ on 
the outward field sweep and ccw on the return sweep. Thus, the origin of the $360^{\circ}$ DWLs observed here is similar to that of previous reports of the rotational demagnetisation of single films [38] and multilayers [39], where an external rotating field imposes a consistent sense of rotation. In the present study, the likely origin of the required asymmetry in rotation directions is the presence of an easy axis / exchange bias dispersion, which we infer from the observation of enhanced magnetic ripple contrast at remanence in those regions where DWLs nucleated. These local variations modulate the alignment of the exchange bias axis and the external magnetic field, which is sufficient to break symmetry and make rotation in one direction energetically more favourable than in the other. Indeed, the magnetisation dispersion for the S2 stacks studied here was surprisingly large. Analysis of Fourier transforms of Fresnel images at remanence (not shown) indicate the dispersion of the S2 stacks to be comparable to those of S1 films, even though the S2 free layer is expected to be significantly constrained by the underlying pinning layers. This local variation in easy axis direction would also account for noticeable lags in the reversal of different regions of the films in figure $6(\mathrm{~g})$ and has been implicated in previous studies of $360^{\circ}$ DWs in simpler systems [38]. An additional effect to consider here is that the easy axis dispersion may also be increased by enhanced ferromagnetic coupling of the free and pinned layers across the tunnel barrier, as suggested by the TEM and EELS analysis, which showed variations in the effective barrier thickness.

A second requirement for DWL nesting is the presence of pinned spins, which most likely arise from static structural defects. The cross-sectional electron microscopy images clearly indicate interfacial roughness throughout the S2 stacks, which is known to influence magnetisation reversal, in particular the formation and annihilation of $360^{\circ}$ DWs. Indeed, artificially-created nanodefects have previously been used for DW pinning [34]. Our data suggest the presence of pinholes within the barrier layer; these would produce ferromagnetic bridges across the barrier and act as pinning centres during the magnetic reversal processes.

In summary, we have demonstrated the formation of remarkably stable concentric $360^{\circ} \mathrm{DW}$ loops in a MTJ film when a magnetic field is applied along the direction perpendicular to the exchange bias. They remain stable up to high applied magnetic fields ( $90 \mathrm{mT}$ ) compared with the coercivity of the free layer. EELS analysis indicated that layer roughness and intermixing of $\mathrm{Fe}$ and $\mathrm{Co}$ into the $\mathrm{MgO}$ could account for the DW pinning and an easy axis dispersion that induces a preferential rotation of magnetisation in different regions of the film. Further investigation is required to determine the possibility of engineering this phenomenon. Whilst DW nesting is expected to impair the smooth operation of devices that rely on simple bistable MTJ switching, it also brings the fascinating prospect of new functionality through multi-state hysteretic switching. 


\section{Acknowledgements}

This work was funded by EU's 7th framework Program IFOX (NMP3-LA-2010 246102)

\section{References}

[1] Slaughter J M 2009 Annu. Rev. Mater. Res. 39, 277.

[2] Zhu J-G 2009 Proc. IEEE 91, 687.

[3] Freitas P P, Ferreira R, Cardoso S and Cardoso F 2007 J. Phys.: Condens. Matter 19, 165221.

[4] Parkin S S P, Jiang X, Kaiser C, Panchula A, Roche K and Samant M 2003 Proc. IEEE 91, 661.

[5] Sugahara S and Nitta J 2010 Proc. IEEE 98, 2124.

[6] Ney A, Pampuch C, Koch R and Ploog K H 2003 Nature 425, 485.

[7] Wolf S A, Awschalom D D, Buhrman R A, Daughton J M, von Molnár S, Roukes M L, Chtchelkanova A Y and Treger D M 2001 Science 294, 1488.

[8] Yuasa S and Djayaprawira D D 2007 J. Phys. D: Appl. Phys. 40, R337.

[9] Hehn M, Lacour D, Montaigne F, Briones J, Belkhou R, El Moussaoui S, Maccherozzi F and Rougemaille N 2008 Appl. Phys. Lett. 92, 072501.

[10] Boeve H, Esparbe L, Gieres G, Bär L, Wecker J and Brückl H 2002 J. Appl. Phys. 91, 7962.

[11] Shang P, Hogwood A, Petford-Long A and Anthony T C 2001 J. Appl. Phys. 89, 7368.

[12] Kovács A, Kohn A, Dean J, Schrefl T, Zeltser A and Carey M J 2009 IEEE Trans. Magn. 45, 3873.

[13] Gogol P, Chapman J N, Gillies M F and Vanhelmont F W M 2002 J. Appl. Phys. 92, 1458.

[14] Schäfer R, Chumakov D, de Haas O, Schultz L, Maass W, Barholz K-U and Mattheis R 2003 IEEE. Trans. Magn. 39, 2089.

[15] Gillies M F, Chapman J N and Kools J C S 1995 J. Appl. Phys. 78, 5554.

[16] Cho H S, Hou C, Sun M and Fujiwara H 1999 J. Appl. Phys. 85, 5160.

[17] Puchalska I and Nicdoba H 1991 IEEE Trans. Magn. 17, 3579.

[18] Heyderman L J, Niedoba H, Gupta H O and Puchalska I B 1991 J. Magn. Magn. Mater. 96, 125.

[19] Jang Y, Bowden S R, Mascaro M, Unguris J and Ross C A 2012 Appl. Phy. Lett. 100, 062407.

[20] Bickel J, Smith S and Aidala K 2014 J. Appl. Phys. 115, 17D135.

[21] Goldman A, Licht A S, Sun Y, Li Y, Pradhan N R, Yang T, Tuominen M T and Aidala K E 2012 J. Appl. Phys. 111, 07D113.

[22] Gonzalez Oyarce A L, Trypiniotis T, Roy P E and Barnes C H W 2013 Phys. Rev. B 87, 174408 .

[23] Nam C and Ross C A 2013 J. Appl. Phys. 113, 123910.

[24] Pradhan N, Licht A, Li Y, Sun Y, Tuominen M and Aidala K 2011 Nanotechnology 22, 485705.

[25] Zhang S, Mu C, Zhu Q, Zheng Q, Liu X, Wang J and Liu Q 2014 J. Appl. Phys. 115, 013908

[26] Geng L and Jin Y M 2012 J. Appl. Phys. 112, 083903.

[27] Liedke M O, Potzger K, Bothmer A H, Fassbender J, Hillebrands B, Rickart M and Freitas P P 2006 J. Appl. Phys. 100, 043918.

[28] Mühlbauer S, Binz B, Jonietz F, Pfleiderer C, Rosch A, Neuebauer A, Georgii R, Boni P 2009 Science 323, 915.

[29] Diegel M, Glathe S, Mattheis R, Scherzinger M and Halder E 2009 IEEE Trans. Magn. 45, 3792.

[30] Kurt H, Oguz K, Niizeki T and Coey J M D 2010 J. Appl. Phys. 107, 083920.

[31] Khamsehpour B, Wilkinson C D W, Chapman J N, Johnston A B 1996 J. Vac. Sci. Technol. B 143381. 
[32] Chapman J N 1984 J. Phys. D: Appl. Phys. 17, 623.

[33] Overwijk M H F, van den Heuvel F C, Bulle-Lieuwma C W T 1993 J. Vac. Sci. Technol. B 11, 2021.

[34] Craig B R, McVitie S, Chapman J N, O’Donnell D O, Johnston A B 2007 J. Phys. D: Appl. Phys. 40, 3991.

[35] Parkin SSP, Kaiser C, Panchula A, Rice P M, Hughes B, Samant M and Yang S-H 2004 Nature Materials 3, 862.

[36] Craven A J, Wilson J A and Nicholson W A P, Ultramicroscopy 92, 165.

[37] Egerton R F, Wang F, Malac M, Moreno M S, Hofer F 2008 Micron 39, 642.

[38] Cohen M S 1963 J. Appl. Phys. 34, 1221.

[39] Heyderman L J, Chapman J N and Parkin S S P 1994 J. Phys. D: Appl. Phys. 27, 881. 

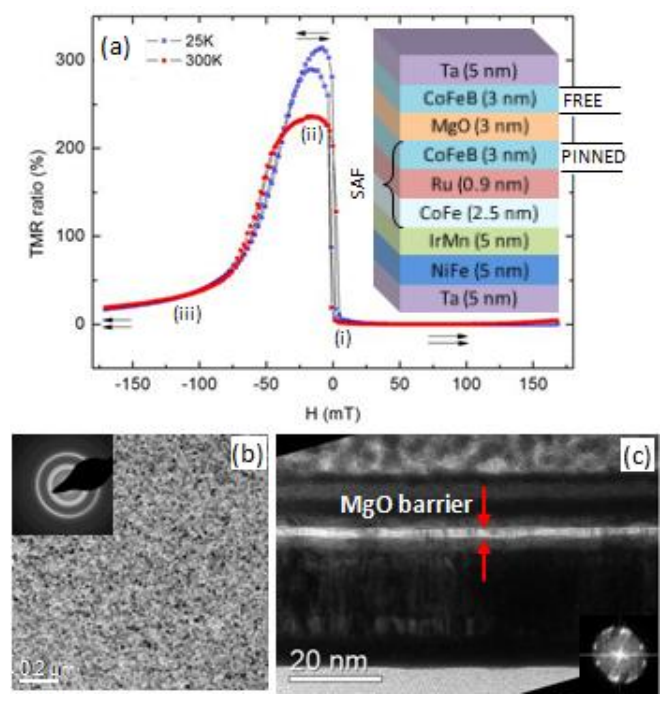

Figure 1. (a) Tunnel magnetoresistance measurements of an S2 stack with (inset) a schematic of the stack structure [38]. Arrows indicate the orientation of the free and pinned CoFeB layers. (b) Plan-view bright field image of a typical MTJ. The contrast variations and characteristic ring structure of the diffraction pattern (inset) indicate the polycrystalline nature of the film. (c) Bright field cross-sectional image of an MTJ with the barrier highlighted. (Inset) A Fourier transform of the image shows distinct spots, indicating crystallinity and texturing. 

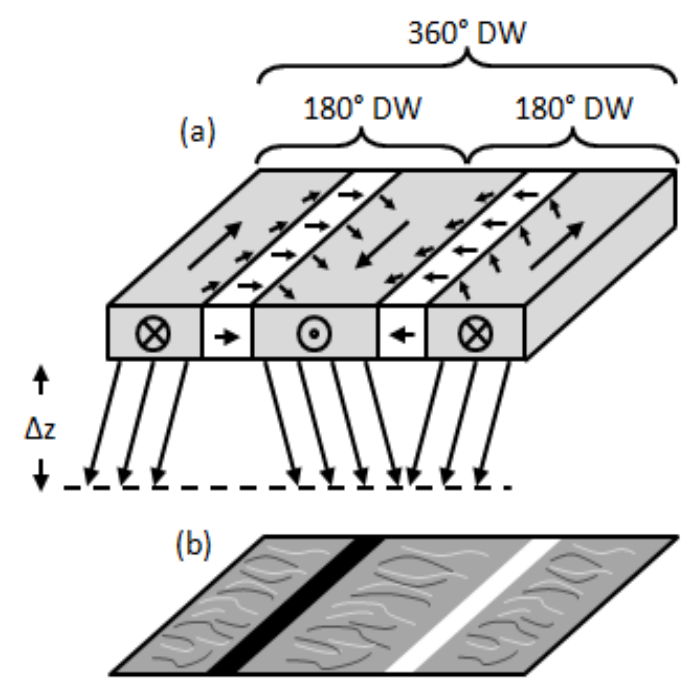

Figure 2. (a) Schematic spin structure of a typical $360^{\circ}$ domain wall, comprising two $180^{\circ}$ Néel domain walls of the same chirality in close proximity. The lower arrows indicate the trajectories of Lorentz-deflected electrons during imaging in the electron microscope. (b) Imaging the sample at a defocus $\Delta \mathrm{z}$ (Fresnel mode) gives rise to a characteristic set of black and white lines in the image. Magnetic ripple contrast also appears within the domains as the result of different anisotropy orientations of the individual crystallites. 

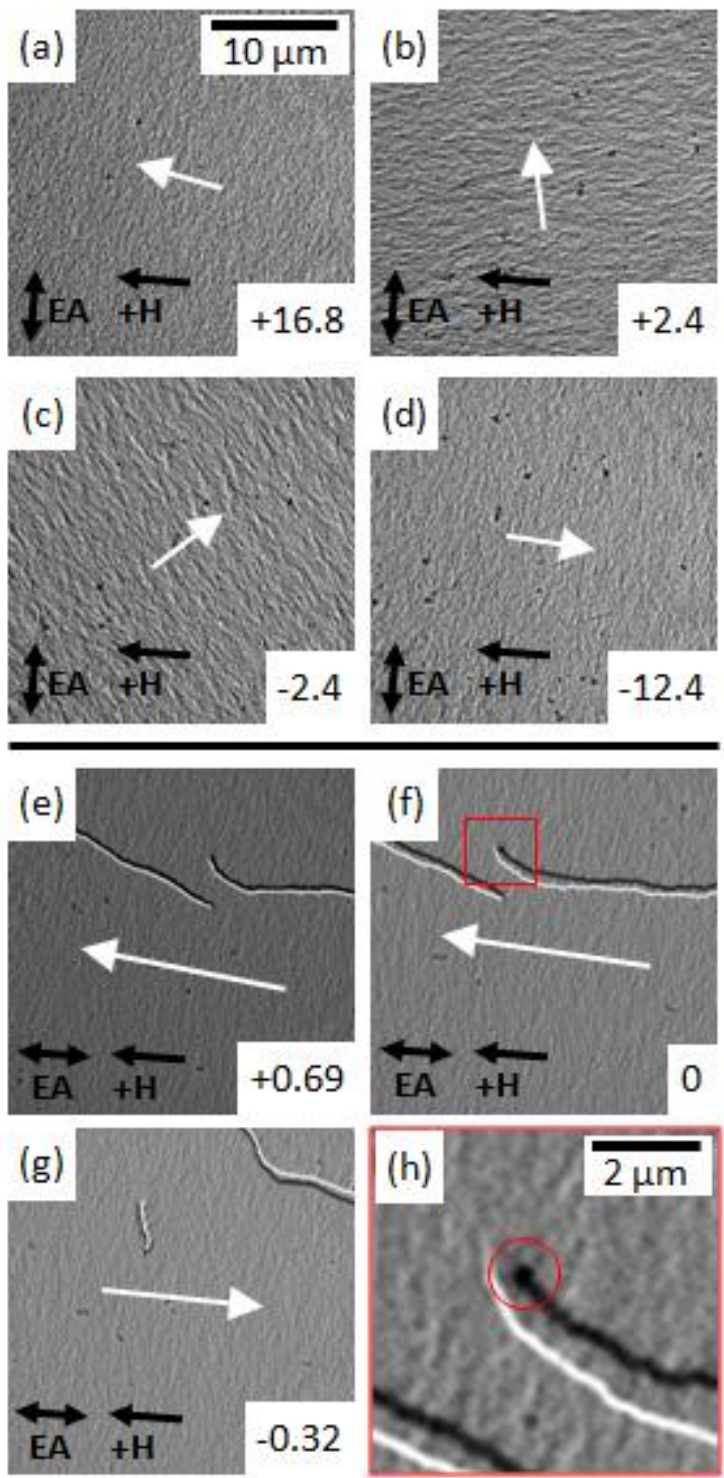

Figure 3. Fresnel image sequence of the reversal of a $\mathrm{Ta} / \mathrm{CoFeB} / \mathrm{MgO} / \mathrm{Ta}$ (S1) film. (a-d) Coherent rotation of magnetisation is observed when the magnetic field is applied perpendicular to the easy axis. (e-g) Easy axis reversal occurs by domain nucleation and growth, with domain walls occasionally pinned by visible defects, such as that highlighted by the red circle in (h), a close-up view of the region enclosed by the red box in (f). The inset numbers indicate the applied field in $\mathrm{mT}$ and the scalebar is common to all but the final image. 

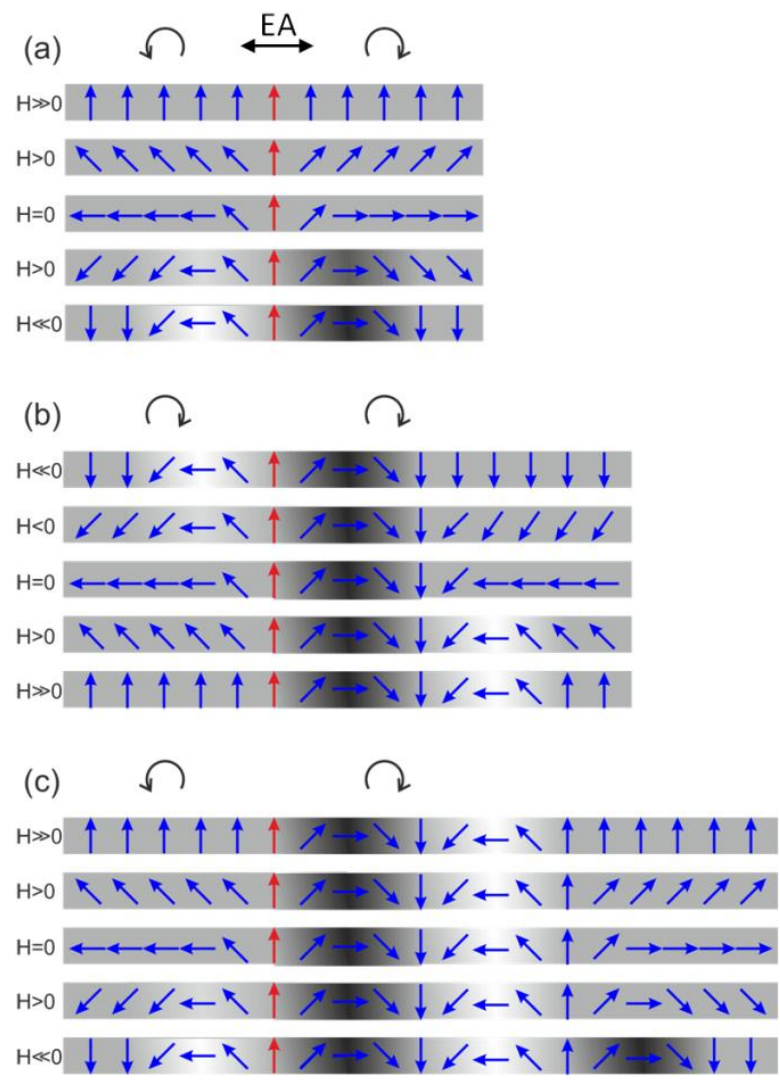

Figure 4. Schematic illustrations of the (a) formation and (b) evolution of $360^{\circ} \mathrm{DWs}$ during magnetisation reversal about a pinned spin (shown in red) and in the presence of a high easy axis dispersion. A second $360^{\circ}$ DW forms during (c), the second field sweep sequence. Each horizontal line of spins illustrates the in-plane spin direction with the external field indicated at the left. Spins respond to the external field direction but are constrained by exchange coupling to their neighbours. There is an easy axis lying along each strip so that spins tend to lie horizontally at remanence. Circular arrows at the top of each set of panels indicate the sense of rotation imposed by the field sweep and easy axis dispersion, with spins to the right of the pinned spin consistently rotating clockwise. Black/white shading indicates the contrast in Lorentz microscopy. 


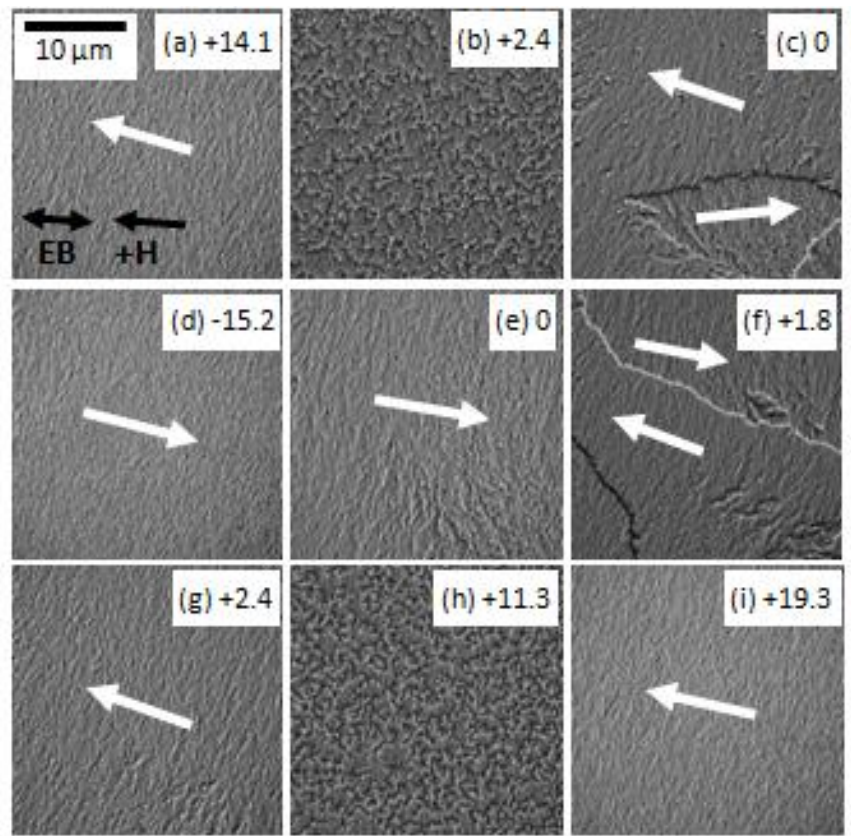

Figure 5. A Fresnel image sequence illustrating the magnetic reversal of the S2 MTJ with a magnetic field applied parallel to the EB axis. Reversal of the free layer occurs via the nucleation and growth of reverse domains which rapidly sweep across the film. The high density contrast observed in images (b) and (h) is attributed to reversal of one of the exchange bias layers, either NiFe or CoFe. The numbers indicate the field values in $\mathrm{mT}$. 

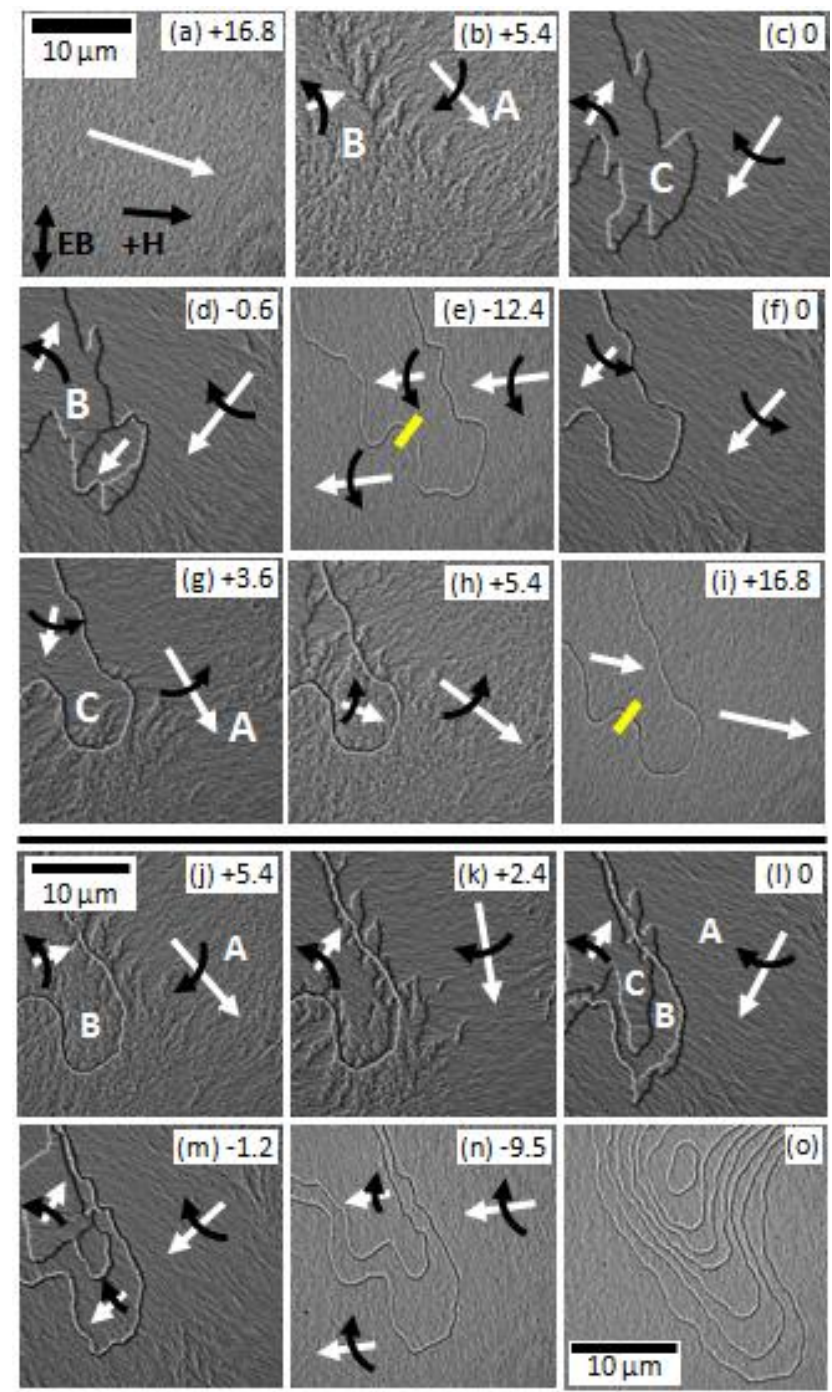

Figure 6. Fresnel image sequence showing the evolution of domain wall loops due to the reversal of the $\mathrm{S} 2$ free layer with a magnetic field applied perpendicular to the EB axis for (ai) the first field sweep and (j-n) a second field sweep. (o) After several field sweeps, 7 nested DWLs were observed elsewhere in the film. Annotated numbers refer to the field strength in $\mathrm{mT}$ at each stage and the scalebar and axis annotations of the first panel are common to all images. As the external field in-plane is applied by tilting the sample, slight shifts in the field of view between successive images are inevitable (see text for details). 


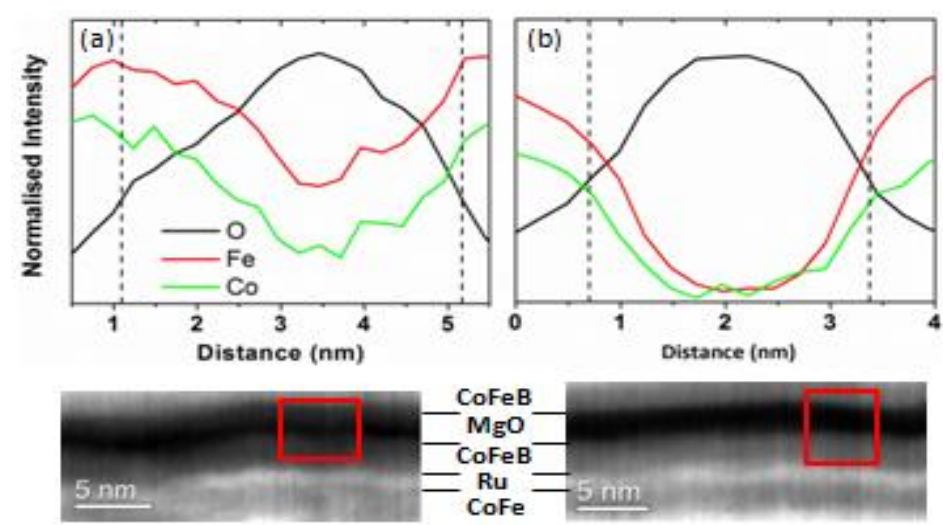

Figure 7. Elemental mapping of two cross-sections through the tunnel barrier of a typical S2 device. (a), (b) Intensity profiles of $\mathrm{O}, \mathrm{Fe}$ and $\mathrm{Co}$ across the $\mathrm{MgO}$ barrier in the MTJ as determined by electron energy loss spectroscopy in two different regions of the film, as indicated by $(\mathrm{c}, \mathrm{d})$ the HAADF images below. Each linetrace is normalised to show the relative rather than absolute variations in composition. 